\title{
General Entropy-Based Framework for a Robust and Fail-Safe Multi-Sensor Data Fusion ${ }^{+}$
}

\author{
Khoder Makkawi ${ }^{1,2}$, Nourdine Ait-Tmazirte ${ }^{3}$, Maan El Badaoui El Najjar ${ }^{4}$ and Nazih Moubayed ${ }^{5}$ \\ 1 CRIStAL-Centre de Recherche en Informatique Signal et Automatique de Lille, Villeneuve-d'Ascq, France \\ 2 Azm Center for Research in Biotechnology and its Application, EDST - Lebanese University, Tripoli, Lebanon \\ 3 Institut de Recherche Technologique Railenium, Famars, France \\ 4 CRIStAL - Centre de Recherche en Informatique Signal et Automatique de Lille, Université de Lille, CNRS, \\ UMR 9189, Lille, France \\ 5 CRSI LaRGES, Lebanese University, Tripoli, Lebanon \\ + Presented at the Entropy 2021: The Scientific Tool of the 21st Century, 5-7 May 2021; Available online: \\ https://sciforum.net/conference/Entropy2021/.
}

Published: 5 May 2021

The 21st century will be the one that will undoubtedly mark the advent of data as a new digital gold. Intelligence is found everywhere in ever-smaller sensors that we no longer perceive. In this study, we are interested in the autonomous vehicle application, vehicles become intelligent, communicating together and with infrastructures. Data is there, everywhere, and constitutes an immaterial source that allows us to increase or even to delegate our decision-making power.

But for certain critical applications from a safety point of view, such as autonomous driving, a decision-based on intentional or non-intentional false, partial or incoherent knowledge could induce dangerous actions having negative effects on goods or people. The delegation of decision-making power to such safety-relevant applications makes regulatory authorities reluctant. It is for these types of applications that the concept of Fault-Tolerant Fusion (FTF) is developed. Being able to detect inconsistencies, while implementing a mitigation strategy (discard or compensation) makes it possible to almost continuously ensure state estimation, and therefore makes at each instant appropriate decisions/action, with a high level of integrity.

In this study, we present a general entropy-based framework for the development of a robust and fail-safe multi-sensor data fusion. From the informational form of the robust used stochastic filter, the MCCUIF (Maximum Correntropy Criterion Unscented Information Filter), to the adaptive diagnostic (FDI: Fault Detection and Isolation) layer based on $\alpha$-Rényi divergence, passing through optimized thresholding (making it possible to maximize the availability of the system while ensuring the high required level of safety), this framework is an efficient and powerful tested example of FTF. It has been tested in real-time and with real data for the high-integrity ego-localization of a vehicle using GNSS and odometer measurements.

(C) 2021 by the authors. Licensee MDPI, Basel, Switzerland. This article is an open access article distributed under the terms and conditions of the Creative Commons Attribution (CC BY) license (http://creativecommons.org/licenses/by/4.0/). 